\author{
Institut für Neutronenphysik \\ KERNFORSCHUNGSANLAGE JULICH \\ des Landes Nordrhein-Westfalen-e.V.
}

\title{
Streuung langsamer Neutronen an polykristallinem Selen und Tellur
}

von

A. Axmann und W. Gissler 
Berichte der Kernforschungsanlcige Jülich - Nr. 479

Institut für Neutronenphysik Jül - 479 - NP

\author{
DOK.: Selenium - Slow Neutron Scattering \\ Tellurium - Slow Neutron Scattering
}

DK.: $\quad 546.23: 539.125 .5 .162 .2: 539.171 .4$

$546.24: 539.125 .5 .162 .2: 539.171 .4$

Zu beziehen durch: ZENTRALBIBLIOTHEK der Kernforschungsanlage Jülich Jülich, Bundesrepublik Deutschland 
phys. stat. sol. 19, 721 (1967)

Subject classification: $6 ; 22.1 .3$

Institut für Neutronenphysik der Kernforschungsanlage Jülich

\title{
Streuung langsamer Neutronen an polykristallinem Selen und Tellur
}

\author{
Von \\ A. Axmann und W. Gissler
}

Mit Hilfe eines Drehkristall-Flugzeitspektrometers wurde die inelastische Streuung langsamer Neutronen an polykristallinem Selen und Tellur untersucht. Es wurden verschiedene Bandenschwerpunkte gefunden, die in ihrer Lage gut mit den durch Infrarotmessungen [1] ermittelten übereinstimmen, jedoch ist ihre Anzahl kleiner. Dieser Umstand zeigt, daß die neutronenspektroskopisch nicht beobachteten Übergänge von Kombinationen herrühren. Bezüglich des Tellurspektrums ergibt sich eine gute t'bereinstimmung mit der Theorie von Hulin [4]. Die Neutronenspektren der beiden isomorphen Kristalle sind einander ähnlich.

The inelastic scattering of slow neutrons by polycrystalline selenium and tellurium is studied using a rotating crystal spectrometer. The frequencies of the observed transitions are consistent with the results of infrared spectroscopy $[1]$, but the number of transitions is lower, which indicates that many of the infrared transitions are combinations. The tellurium spectrum is in good agreement with Hulin's theory [4]. The neutron spectra of the two isomorphous lattices are similar.

\section{Gittersehwingungen in Selen und Tellur}

Obgleich Selen und Tellur bezüglich ihrer Halbleitereigenschaften eingehend untersucht wurden, ist der Erforschung ihres gitterdynamischen Verhaltens vergleichsweise nur wenig Aufmerksamkeit geschenkt worden.

Selen und Tellur sind isomorph und kristallisieren in einem hexagonalen Dreipunktgitter entsprechend Fig. 1. Die Atome sind auf Spiralen angeordnet, deren Achsen parallel zur hexagonalen $C$-Achse verlaufen. Die Bindung der auf einer Spirale liegenden Atome ist kovalent und stärker als die teils van der Waalsteils metallische Bindung von Atomen verschiedener Spiralen.

Von Caldwell und Fan [1] liegen für Selen und Tellur Infrarot-Transmissionsmessungen vor, die Aufschlüsse über die optischen Schwingungsbranchen im Zentrum der Brillouin-Zone ergeben. Die Interpretation dieser Messungen durch Boitsov [2 und 3] und durch Hulin [4] ist jedoch teilweise widersprechend.

Wegen der schwachen Wechselwirkung der Atome auf verschiedenen Spiralen legte Boitsov seinen Rechnungen das Modell einer Spirale ohne Wechselwirkung mit den Nachbarspiralen zugrunde. Die Kraftkonstanten des Modells wurden den optisch bestimmten Übergangsfrequenzen angepaßt. Bei diesem Verfahren interpretiert Boitsov die experimentell bestimmten Übergänge als direkte Übergänge, obwohl Auswahlregeln einige dieser Übergänge - zumindest jn sehr reinen Kristallen - verbieten müßten. Für Selen konnte eine gute Kon- 

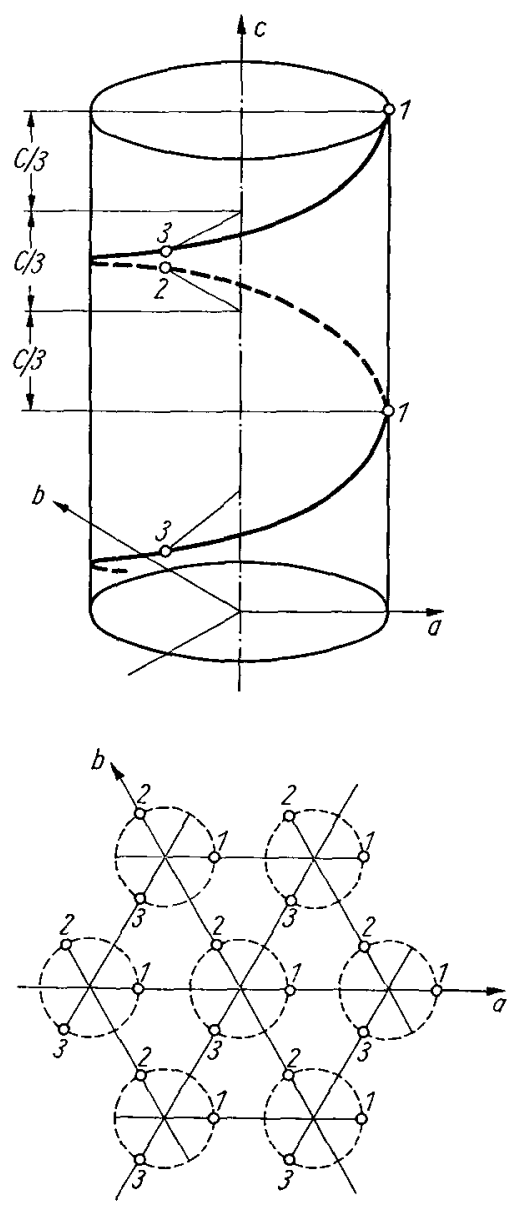

Tig. 1. Anordnung der selen- bzw. Telluratome in der hexagonalen Gitterzelle

sistenz des errechneten mit dem experimentellen Spektrum erzielt werden; bei Tellur ergaben sich besonders bei niedrigen Frequenzen größere Abweichungen.

Hulin schlug für Tellur ein verfeinertes dreidimensionales Modell vor, welches die Wechselwirkung verschiedener Spiralen berücksichtigt. Im Gegensatz zu Boitsov paßt er die Kraftkonstanten seines Modells den elastischen Konstanten an. Dadurch werden diese nicht von einer a priori-Interpretation der optisch bestimmten Übergänge abhängig. Hulin findet nur eine Utbereinstimmung mit den ersten beiden optisch bestimmten Übergängen, dagegen erklärt er höherenergetische Übergänge nicht als direkte Ubergänge, sondern unter Berücksichtigung von Auswahlregeln als Kombinationsübergänge.

Die inelastische Neutronenstreuung sollte weiteren Aufschluß über die Gitterdynamik liefern; insbesondere sollte sie in der Lage sein, die Natur der optischen Übergänge aufzuklären: 1. Hier gelten nicht die für Infrarotmessungen gültigen Auswahlregeln. 2. Der Anteil von Multiphononprozessen ist weitaus kleiner als bei Infrarotmessungen.

\section{Inelastische Neutronenstreuung an Polykristallen}

Da Einkristalle der vorzugsweise kohärenten Streuer Selen und Tellur nur schwer erhältlich sind, haben wir Pulverproben benutzt. Die hierbei durch die Streuung gelieferten Informationen sind geeignet gebildete Mittelwerte über die verschiedenen Richtungen der Mikrokristalle im Pulver. Der kohärent inelastische Streuquerschnitt $\left(\partial^{2} \sigma / \partial \Omega \partial \omega\right)$ für Polykristalle wurde von Egelstaff [5] angegeben :

$$
\frac{\partial^{2} \sigma}{\partial \Omega \partial \omega}=\frac{\sigma_{\text {coh }}}{4 \pi} I Z
$$

$I$ stellt im wesentlichen die inkohärente Streufunktion dar:

$$
I=\frac{\hbar}{2 m} \mathrm{e}^{-2 W} \frac{k^{\prime}}{k_{0}} \sum_{i} T\left(\omega_{i}\right) \frac{\varkappa^{2}}{\omega_{i}} \cos ^{2} \vartheta_{i} q_{i}^{2}\left(\frac{\mathrm{d} q_{i}}{\mathrm{~d} \omega_{i}}\right) \text {. }
$$

Der Faktor $Z$ berücksichtigt, daß bei der kohärenten Streuung die Impulserhaltungsbedingung $\delta(\boldsymbol{x}+\boldsymbol{q}-2 \pi \boldsymbol{\tau})$ erfüllt werden muß; beim Polykristall muß diese Bedingung über alle Orientierungen von $\varkappa$ und $q$ bezüglich der Richtung 
von $2 \pi \tau$ gemittelt werden. Dies ergibt

$$
Z=\left\{\begin{array}{cl}
\frac{\pi F_{\tau}}{2 B \varkappa q \tau} & \text { für } 2 \pi|\boldsymbol{\tau}|-|\boldsymbol{q}| \leqq|\boldsymbol{x}| \leqq 2 \pi|\boldsymbol{\tau}|+|\boldsymbol{q}| \\
0 & \text { im übrigen Bereich. }
\end{array}\right.
$$

Gleichung (2) und (3) sind unter der Voraussetzung isotroper Dispersionsbeziehungen $\omega=\omega(|\boldsymbol{q}|)$ gültig. Hier verwenden wir jedoch Gleichung (3) näherungsweise auch für nichtisotrope Dispersionsbeziehungen.

Die in (1), (2) und (3) verwendeten Symbole haben folgende Bedeutung: $\sigma_{\text {con }}$ ist der kohärente Streuquerschnitt, $m$ die Masse des Streukerns, $2 W$ der Debye-Waller-Faktor, $k^{\prime}$ bzw. $k_{0}$ die Wellenzahl des gestreuten bzw. des einfallenden Neutrons, $\hbar \varkappa$ ist der Impuls- und $\hbar \omega$ der Energieübertrag der Neutronen bei der Streuung. Der Index $i$ bezeichnet eine der drei Polarisationsrichtungen. $F_{\tau}$ ist die Summe der Strukturfaktoren aller Netzebenen mit dem Netzebenenabstand $d=1 /|\boldsymbol{\tau}| . \quad T(\omega)=1 /[\exp (\hbar \omega / k T)-1]$ ist die Besetzungszahl und $\vartheta_{i}$ der Winkel zwischen Polarisationsvektor und $x$; es gilt $\cos \vartheta_{i}=$ $=\left\{(2 \pi \boldsymbol{x})^{2}-\boldsymbol{x}^{2}-\boldsymbol{q}^{2}\right\} /(2|\boldsymbol{x}||\boldsymbol{q}|) . B$ ist das Volumen der Gitterzelle. Die Bereiche, in denen Streuung möglich ist, sind nach (3) durch die von $|\boldsymbol{\varkappa}|=2 \pi|\boldsymbol{\tau}|$ (Lagen der Debye-Scherrer-Reflexe) ausgehenden Dispersionskurven $\pm q(\omega)$ gegeben. In Fig. 2 sind diese Bereiche für einzelne isotrope akustische und optische Dispersionskurven qualitativ dargestellt. Die sich für Messungen mit konstantem Streuwinkel $\delta$ auf Grund von Energie- und Impulssatz ergebenden Wege sind in der $\varkappa, \omega$-Ebene für verschiedene $\delta$ gestrichelt eingezeichnet.

Da die Streuintensität nach (2) und (3) proportional zu $q(\mathrm{~d} q / \mathrm{d} \omega)$ ist, erhalten wir Intensitätsmaxima bei den Energien der optischen Zweige und in der Nähe der Grenzschwingungen der akustischen Zweige. Die Intensität für Übergänge an den optischen $Z$ weigen ist am größten, wenn der betreffende Streubereich durch Meß,,wege" in der Nähe von $|x| \approx$ $\approx 2 \pi|\boldsymbol{\tau}|$ untersucht wird, da hier Energieund Impulssatz für die meisten Phononen zu erfüllen ist.

Auf Grund von (3) erwarten wir an den Grenzen der Bereiche möglicher Streuung scharfe Intensitätskanten für die longitudinalen Moden $(\cos \vartheta=1$ ) der akustischen Zweige. Cocking und Guner [6] sowie Larsson et al. [7] haben diese Intensitätskanten an polykristallinem Zinn bzw. Aluminium angedeutet gefunden. Im besonders günstig gelagerten Fall des Argons konnten Kroó et al. [8] aus der Lage dieser Intensitätskanten eine ,mittlere" Dispersionskurve gewinnen.

In vielen Fällen, wie hier bei Selen und Tellur, wird dies jedoch nur schlecht

Fig. 2. Bereiche möglicher streuung in der $x, \omega$-Ebene. Die gestrichelten Kurven stellen die für konstante Streuwinkel müglichen "Wege“ in der $\varkappa$, $\omega$-Hbene dar. Schnittpunkte dieser Linien mit den Grenzen der Streubereiche ergeben für longitu-

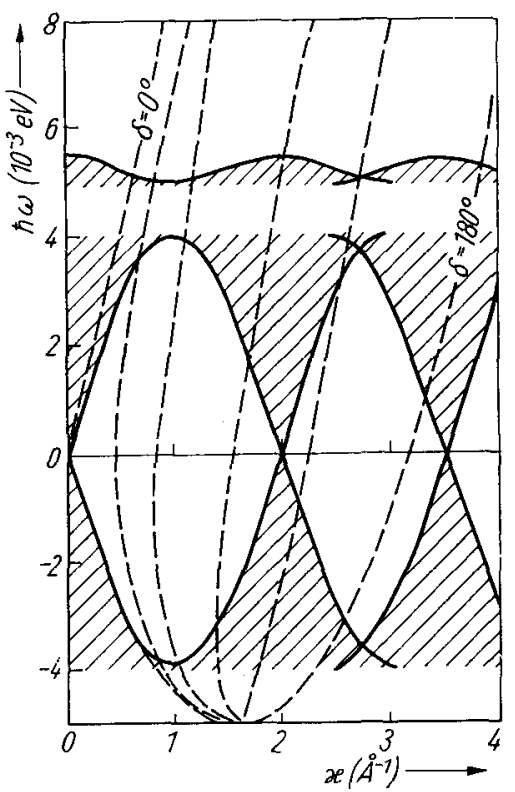
dinale Moden Intensitätsmaxima 
möglich sein. Bei großer Anisotropie der Kristalle bilden die den verschiedenen Kristallrichtungen zugehörigen Dispersionskurven ein breites Band, was zu einer Verschmierung der Intensitätskanten führt. Außerdem wirkt sich erschwerend aus, daß bei weniger symmetrischen Gittern als dem kubisch flächenzentrierten mangels Auslöschungen und kleinerer Multiplizitäten die DebyeScherrer-Reflexe dichter liegen und ihre Intensitäten kleiner sind. Dieser Umstand bewirkt eine Überlappung der Bereiche möglicher Streuung, so daß im Spektrum Intensitätskanten sich so überlagern können, daß deren Auflösung nicht möglich ist.

\section{Messungen und Resultate}

Die Messungen wurden mit einem Drehkristall-Flugzeitspektrometer am FRJ-2 Reaktor in Jülich durchgeführt. Fig. 3 zeigt die schematische MeBanordnung. Der durch einen totalreflektierenden Neutronenleiter geführte subthermische Neutronenstrahl (gefiltert mit einem gekühlten Be-Kristall) wird von einem sich schnell drehenden Aluminium-Einkristall (ca. $10000 \mathrm{U} / \mathrm{min}$ ) monochromatisiert und in kurze Pulse zerhackt. Die an der Probe gestreuten Neutronen werden auf zwei 3,20 m langen Strecken einer Flugzeitanalyse unter-

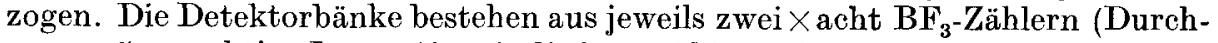
messer $5 \mathrm{~cm}$, aktive Länge $40 \mathrm{~cm}$ ), die in zwei hintereinanderliegenden Schichten angeordnet sind. Die Energieauflösung der einfallenden Neutronen betrug $\Delta E=1,5 \times 10^{-4} \mathrm{eV}$. Die Energieauflösung der gestreuten Neutronen betrug für $E=5 \times 10^{-3} \mathrm{eV}$ (elastische Streuung) $\Delta E=3 \times 10^{-4} \mathrm{eV}$ und für $E=$ $=25 \times 10^{-3} \mathrm{eV} \Delta E=2 \times 10^{-3} \mathrm{eV}$.

Durch eine Pulver-Röntgenaufnahme von Selen bzw. Tellur haben wir uns davon überzeugt, daß keine andere als die hexagonale Phase in merkbarer Konzentration vorlag. Die Reinheit der Proben betrug nach Angaben der Lieferfirma 99.9\%. Die Pulverproben

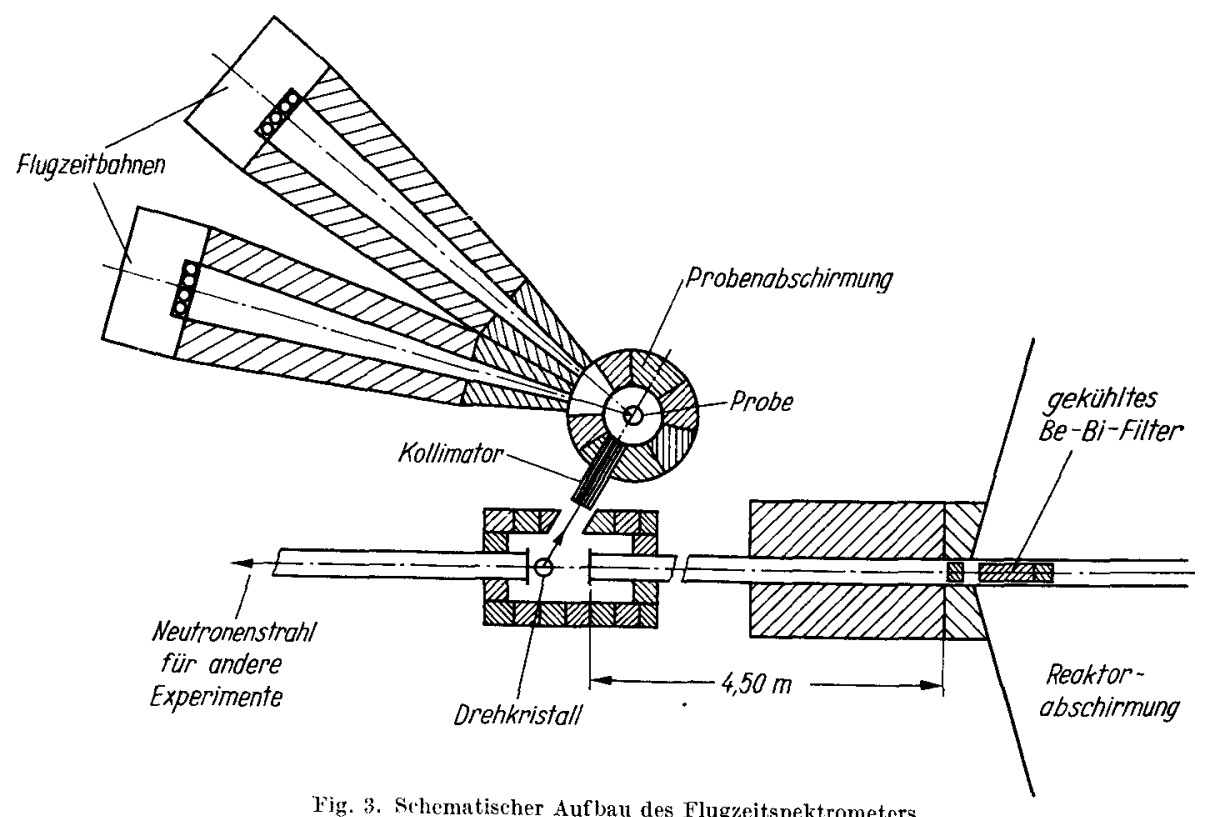

Fig. 3. Schematischer Aufbau des Flugzeitspektrometers 
Fig. 4. Flugzeitspektren von Selen und Tellur für Streuwinkel von 80 und $105^{\circ}$. Die gestrichelten Kurven stellen den erechneten Beitrag der Mehrphononen-

wurden in eine $1 \mathrm{bzw} .2 \mathrm{~cm}$ dicke plattenförmige Aluminium küvette (Wandstärke $1 \mathrm{~mm}$ ) eingefüllt. Aus der gemessenen Transmission, dem bekannten $\mathrm{Ab}$ sorptionsquerschnitt bei $5 \times 10^{-3}$ $\mathrm{eV}$ für Selen von $23,2 b \mathrm{bzw}$, für Tellur von $10 b[9]$ und der jeweils experimentell bestimmten Pulverdichte ergab sich für die Selenprobe eine 12- für die Tellur. probe ein 13prozentige Streuwahrscheinlichkeit. Zweifachstreuprozesse sind damit, wie eine einfache Abschätzung zeigt, zu vernachlässigen.

Fig. 4 zeigt die gemessenen Flugzeitspektren für Streuwinkel von 80 und $105^{\circ}$ für Selen bzw. Tellur. Die Messungen sind bereits bezüglich Untergrund korrigiert und auf die gleiche Monitorrate bezogen. Es wurden jeweils zwei der 16- $\mu$ s-Meßkanäle zu einem Punkt zusammengefaßt. Die Zeitauflösung ent. spricht für Neutronenenergien $E>10 \times 10^{-3} \mathrm{eV}$ etwa dem

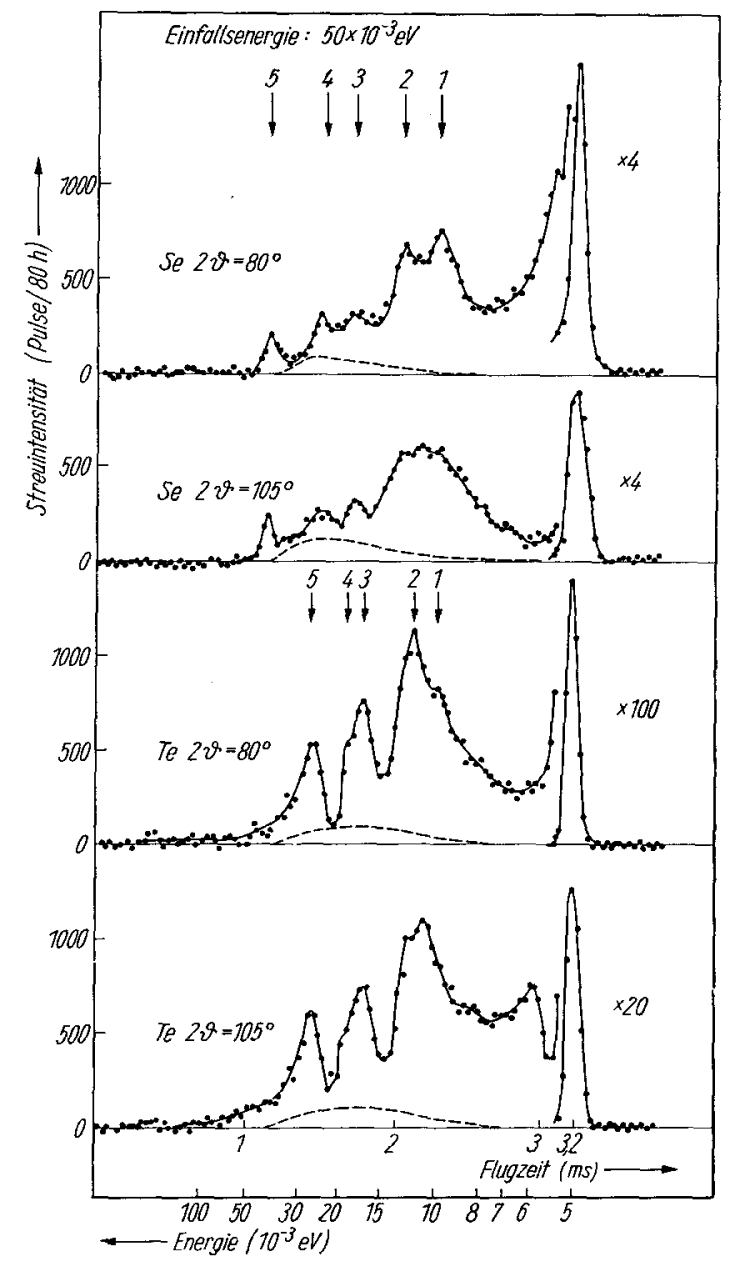
Abstand dreier Meßpunkte.

Das Signal-Untergrund-Verhältnis beträgt im Maximum Nr. 1 ca. 4:1. In diesem Maximum wurden ca. 800 Signalimpulse pro Meßpunkt in vier Tagen ausgezählt.

Neben den Messungen für Streuwinkel von 80 und $105^{\circ}$ wurden an Selen noch weitere für Streuwinkel von $40,50,60$ und $70^{\circ}$, allerdings bei einer um den Faktor 2 schlechteren Auflösung, durchgeführt. Diese Messungen zeigten im wesentlichen die gleiche Struktur wie die der Fig. 3 ; insbesondere ließen diese Meßergebnisse keine Veränderungen der Lage der in Fig. 3 bezifferten Maxima erkennen.

Energieverlustprozesse der Neutronen könnten infolge von ,Zyklusüberlappung" als Maxima auf der hochenergetischen Seite des EnergiegewinnNeutronenspektrums erscheinen. Die Lage dieser Maxima ist jedoch von der Pulsfolgefrequenz der einfallenden Neutronen abhängig. Durch Messung bei zwei verschiedenen Drehzahlen $(120$ und $90 \mathrm{U} / \mathrm{s})$ haben wir uns davon überzeugt, daß keines der von uns beobachteten Maxima der Fig. 4 von der Pulsfolgefrequenz abhängig ist.

47 physica 
Mehrphononenprozesse werden pauschal durch eine effektive Debye-Temperatur $\Theta_{\text {eff }}$ in inkohärenter Näherung nach einem Ausdruck von Sjölander [10] abgeschätzt. Wir setzten dabei für Tellur $\Theta_{\text {eff }}=130^{\circ} \mathrm{K}$ [11] und für Selen den $\sqrt{m_{\mathrm{Te}} / m_{\mathrm{Se}}} \approx 1,3$ fachen Wert ein $\left(m_{\mathrm{Te}}\right.$ bzw. $m_{\mathrm{Se}}$ ist die Masse des Tellur- bzw. des Selenatoms). Die Resultate sind als gestrichelte Linien in Fig. 4 eingezeichnet.

\section{Diskussion}

Die Tabellen 1 und 2 zeigen die Lagen der von uns beobachteten Maxima in $10^{-3} \mathrm{eV}$ abzüglich der Neutroneneinfallsenergie für Selen und Tellur. Zum Vergleich sind die durch optische Messungen [1] bestimmten Übergangsfre. quenzen und die theoretischen Werte für Normalschwingungen von Tellur von Hulin [4] mit angeführt (eine Entzerrung des Flugzeitmaßstabs in den Energiemaßstab unter Berücksichtigung des Temperaturfaktors hatte keinen Einfluß auf die Lage der Maxima).

Tabelle 1

(Selen)

\begin{tabular}{l|c|c|c|c|c|c|c|c|c} 
Maximum Nr. & 1 & 2 & 3 & 4 & 5 & 6 & 7 & 8 & 9 \\
\hline Neutronen-Mess. & 5,1 & 7,8 & 13 & 18 & 35 & - & - & - & - \\
Infrarot-Mess. & - & - & - & 17,5 & 34 & 44 & 56 & 59 & 82
\end{tabular}

Tabelle 2

\begin{tabular}{l|c|c|c|c|c|c|c|c|c|c|c} 
& \multicolumn{10}{c}{ (Tellur) } \\
\hline Maximum Nr. & $\mathbf{1}$ & $\mathbf{2}$ & $\mathbf{3}$ & $\mathbf{4}$ & 5 & 6 & 7 & $\mathbf{8}$ & 9 & 10 & $\mathbf{1 1}$ \\
\hline Neutronen-Mess. & 5,3 & $\mathbf{6 , 6}$ & 11,1 & 13,6 & 20,5 & - & - & - & - & - & - \\
Infrarot-Mess. & - & - & 11,0 & 13,1 & - & 22,5 & 26,2 & 39,7 & 44,3 & 51,0 & $*$ \\
Theorie Hulin**) & - & - & $\mathbf{9}$ & 15 & $(20)$ & - & - & - & - & - & -
\end{tabular}

*) Die Infrarotmessungen für Tellur erstrecken sich nur bis etwa $6 \times 10^{-2} \mathrm{eV}$.

**) Werte für die Grenzschwingung $q=0$.

Phononenübergänge akustischer Zweige: Unsere Meßergebnisse zeigen bei niedrigen Energien zwei je nach Meßwinkel mehr oder weniger gut aufgelöste Maxima (Nr. 1 und 2); wir nehmen an, daß sie von den akustischen Zweigen herrühren. Ihre Lagen stimmen im Falle des Selen gut mit der bekannten DebyeTemperatur $\Theta_{\mathrm{D}}$ überein $\left(K \Theta_{\mathrm{D}}=6,5 \times 10^{-3} \mathrm{eV}\right)$. Messungen bei verschiedenen Streuwinkeln ergaben keine Änderung der Lagen dieser Maxima, obwohl dies nach (2) und (3) zu erwarten wäre. Eine Erklärung hierfür ist wahrscheinlich in dem in Abschnitt 2 beschriebenen Umstand zu suchen, der auf Grund einer starken Anisotropie und einer Utberlappung der Streubereiche die Intensitätskanten verschmiert und die Übergänge in der Nähe der Zonengrenze begünstigt.

Phononenübergänge optischer Zweige: Die Energien der übrigen von uns beobachteten Maxima zeigen innerhalb der Meßfehlergrenzen ebenfalls keine Abhängigkeit vom Streuwinkel. Wir deuten sie als Ubergänge optischer Zweige.

Auf Grund der Isomorphie und ähnlicher Bindungsverhältnisse von Selen und Tellur erwarten wir eine Ähnlichkeit der Spektren beider Substanzen. Tatsächlich konnte diese auch durch die Infrarotmessungen nachgewiesen werden. Sämtliche hierbei beobachteten Ubergangsfrequenzen stehen ungefähr 
im Wurzelverhältnis der Atommassen $\sqrt{\frac{m_{\mathrm{Te}}}{m_{\mathrm{Se}}}} \approx 1,3$. Diese Ähnlichkeit wird auch in dem von uns gemessenen Spektrum hinsichtlich der Maxima 1 bis 5 beobachtet.

Ein Vergleich mit den Infrarotwerten zeigt, daß die von uns gefundenen Übergangsfrequenzen zwar geringer an Zahl sind, jedoch hinsichtlich ihrer Lage sehr gut mit einzelnen Infrarotwerten übereinstimmen. Da bei den Neutronenmessungen Mehrphononenprozesse unter den hier betrachteten optischen Energiebanden vernachlässigbar sind, folgt daraus, daß einige der Infrarotwerte Übergänge sind, die von Mehrphononenprozessen herrühren, was den Annahmen von Boitsov widerspricht.

Nach Hulin entsprechen nur die beiden ersten Infrarotfrequenzen direkten Übergängen, während ein weiterer direkter Übergang zu einem optischen Band bei $20 \times 10^{-3} \mathrm{eV}$ für Infrarotmessungen verboten ist und alle übrigen gemessenen Infrarotfrequenzen durch Mehrfachprozesse erklärt werden. Unsere Messungen zeigen eine gute Übereinstimmung mit diesen Annahmen ${ }^{1}$ ); tatsächlich beobachten wir neben den bereits optisch bestimmten Übergängen 3 und 4 den von Hulin vorausgesagten Übergang bei $20 \times 10^{-3} \mathrm{eV}$. Weitere Übergänge sind nicht festzustellen. ${ }^{2}$ )

Herrn Dr. T. Springer verdanken wir die Anregung der Arbeit und zahlreiche Diskussionen. Herrn Dr. H. Stiller danken wir für seine Unterstützung.

\section{Literatur}

[1] R. C. CaLdwell und H. J. Fav, Phys. Rev. 114, 664 (1959).

[2] V. G. Borrsov, Fiz. tverd. Tela 5, 1050 (1963).

[3] V. G. Borrsov, Fiz. tverd. Tela 5, 1822 (1963).

[4] M. Huurv, Ann. Phys. (France) 8, 647 (1963).

[5] P. A. Egelstaff, AERE Rep. N/R 1164 (1953).

[6] S. J. Cocking und Z. GUNER, Inelastic Scattering of Neutrons in Solids and Liquids, IAEA, Vienna 1963 (S. 237).

[7] K. E. Larsson, U. DahlboRg und D. Jovic, Inelastic Scattering of Neutrons in Solids and Liquids, IAEA, Vienna 1965 (S. 117).

[8] N. Kroó, G. Borgonovi, K. SköLd und K. E. Larsson, Inelastic Seattering of Neutrons in Solids and Liquids, IAEA, Vienna 1965 (S. 115).

[9] D. J. Hughes und R. B. Schwartz, Neutron Cross Sections, U.S. Atomic Ene rgy Commission BNL 325 (1955).

[10] A. Stölandder, Ark. Fys. (Sweden) 17, 315 (1958).

[11] L. GmeLrn, Handbuch der anorganischen Chemie, Teil A (Selen), Verlag Chemie, Weinheim /Bergstr. 1953.

[12] R. GETCK, persönliche Mitteilung.

(Received November 29, 1966)

1) Auch neuere Messungen mit der Infrarot-Reststrahlenmethode von Geick [12] scheinen diese Annahmen zu bestätigen.

2) Bei der Untersuchung von Tellurproben, die Verunreinigungen von Sauerstoff und Schwefel von einigen Gewichtsprozenten enthielten, konnten im Neutronenspektrum bei $40 \times 10^{-2} \mathrm{eV}$ und $80 \times 10^{-2} \mathrm{eV}$ Maxima beobachtet werden. Ihre Natur soll in einer anderen Arbeit geklärt werden.

47* 